\title{
Nerve Root Neoplasm
}

National Cancer Institute

\section{Source}

National Cancer Institute. Nerve Root Neoplasm. NCI Thesaurus. Code C5119.

Benign and malignant neoplasms arising from one or more of the cervical, thoracic, lumbar, sacral, or coccygeal nerve roots. The majority of these tumors are benign. Clinical manifestations may include pain, weakness and loss of sensation along the course of the involved nerve root. Large tumors may cause spinal cord compression. 